\title{
Mass enhancement and magnetic order at the Mott-Hubbard transition
}

\author{
S. A. Carter and T. F. Rosenbaum \\ The James Franck Institute and Department of Physics, The University of Chicago, Chicago, Illinois 60637 \\ P. Metcalf, J. M. Honig, and J. Spalek \\ Department of Chemistry, Purdue University, West Lafayette, Indiana 47907
}

(Received 7 September 1993)

\begin{abstract}
We study the evolution with pressure $P$ and band filling $y$ of the heat capacity, Hall coefficient, and resistivity at the approach to the $T \rightarrow 0$ Mott-Hubbard metal-insulator transition (MIT) in highly correlated $\mathrm{V}_{2-y} \mathrm{O}_{3}$. Under $P$, the electronic effective mass $m^{*}$ diverges at the MIT with a negligible change in carrier concentration $n$ away from half-filling. Conversely, in the doped system $m^{*}$ actually decreases as the MIT is approached, while $n$ increases linearly with $y$. The low- $T$ magnetic order in the metal helps us deconvolute contributions from charge correlations and spin fluctuations.
\end{abstract}

Although rare in most metallic systems, (incipient) antiferromagnetism appears to be a trademark of highly correlated metals, including the heavy fermion compounds, ${ }^{1}$ the high- $T_{c}$ superconductors, ${ }^{2}$ and the MottHubbard systems $\mathrm{V}_{2} \mathrm{O}_{3}$ (Refs. 3 and 4) and $\mathrm{Ni}\left(\mathrm{Se}_{1-x} \mathrm{~S}_{x}\right)_{2} \cdot{ }^{5}$ This underlying magnetism clearly influences the low-temperature behavior of highly correlated materials and, in fact, may be integral to the superconductivity observed in some of them. Hence, the problem of providing a proper theoretical framework for treating spin density fluctuations associated with itinerant electrons together with the properties of local moments in metals is being revisited. A number of efforts have addressed the role of reduced moment antiferromagnetism in heavy fermion materials and local moments in disordered metals. ${ }^{6}$ However, experiments which probe the effect of magnetism on the MottHubbard metal-insulator transition (MIT), where electron-electron interactions are strongly enhanced, are essentially nonexistent.

Models of the Mott-Hubbard MIT predict a transition from the well-known period-doubling antiferromagnetic insulator to a metal with an incommensurate spiral spin density wave (SDW). ${ }^{7}$ Recently, Bao et al. ${ }^{8}$ have observed such a transition with Néel temperature $T_{N} \sim 10$ $\mathrm{K}$ in $\mathrm{V}_{2-y} \mathrm{O}_{3}$, a material which has served as the prototype for Mott-Hubbard physics 9,10 and the BrinkmanRice picture of the correlated metal. ${ }^{11}$ Moreover, inelastic neutron measurements reveal a remarkably robust spin fluctuation spectrum up to $T \sim 10 T_{N}$ at the SDW incommensurate wave vector, similar to what is observed in the high- $T_{c}$ superconductor $\mathrm{La}_{2-x} \mathrm{Sr}_{x} \mathrm{CuO}_{4}{ }^{2}$ This apparent agreement with a class of Mott-Hubbard theories and the striking similarities of aspects of the heavy, reduced-moment antiferromagnetic metal in $\mathrm{V}_{2-y} \mathrm{O}_{3}$ to the metallic state in heavy fermion and cuprate superconductors, makes vanadium sesquioxide an ideal system in which to elucidate the role of magnetism in highly correlated electron systems.

In addition to magnetism, theories of Mott-Hubbard systems have focused on parametrizing the system in terms of the effective mass, $m^{*}$, and the density of carriers, $n .{ }^{12}$ In this paper, we study the $T \rightarrow 0$ MIT in
$\mathrm{V}_{2-y} \mathrm{O}_{3}$ by deconvoluting the behavior of $m^{*}$ and $n$ through Hall coefficient and heat capacity measurements. We find that if we fix the number of magnetic impurities and move across the transition with pressure, then the electronic effective mass diverges at the MIT, even in the presence of magnetic ordering. However, contrary to expectations, the effective mass does not diverge if we move across the transition with doping. Furthermore, we demonstrate that the spin degrees of freedom so strongly affect the properties of the correlated metal that conventional approaches to determining the electronic effective mass give misleading results; only measurements at milliKelvin temperatures $\left(T \ll T_{N}\right)$ accurately reflect the salient physics. Finally, we attempt to reconcile these results within a unified Mott-Hubbard framework.

Single crystals of $\mathrm{V}_{2} \mathrm{O}_{3}$ were grown using a skull meter and were then annealed in a $\mathrm{CO}-\mathrm{CO}_{2}$ atmosphere at $1400^{\circ} \mathrm{C}$ for approximately two weeks to adjust the stoichiometry uniformly. ${ }^{13}$ The stoichiometry of the $\mathrm{V}_{2-y} \mathrm{O}_{3}$ crystals were determined within 0.001 of $y$ using thermograviometric analysis. ${ }^{14}$ The acuity and reproducibility of the cusp in the magnetic susceptibility at the antiferromagnetic transition indicates that the homogeneity is within 0.0005 of $y$. Hall measurements were performed in the linear field regime on crystals with typical dimensions $1 \times 1 \times 0.15 \mathrm{~mm}^{3}$. All resistivity and Hall data were obtained in the Ohmic and frequencyindependent limits using a standard lock-in technique at $16 \mathrm{~Hz}$ for $0.02 \mathrm{~K} \leq T \leq 300 \mathrm{~K}$. Pressure measurements were performed in a $\mathrm{BeCu}$ hydrostatic pressure cell with silicone oil as the pressure medium and a fragment of $\left(\mathrm{V}_{0.99} \mathrm{Ti}_{0.01}\right)_{2} \mathrm{O}_{3}$ as the manometer. ${ }^{15}$ The heat capacity was determined by measuring the exponential decay of $T$ after the application of a known heat pulse. The heat capacity of $\mathrm{V}_{1.987} \mathrm{O}_{3}$ under pressure was ascertained by mounting the heater and a Speer carbon chip thermometer on a $35-\mathrm{mg}$ sample in the $\mathrm{BeCu}$ pressure cell. The teflon bucket of thickness $1.2 \mathrm{~mm}$, which contained the sample and the pressure medium, served as the heat leak. The background $(\sim 40 \%)$ was determined in the low pressure insulating state of $\mathrm{V}_{1.987} \mathrm{O}_{3}$, for which the lowtemperature specific heat is known to be negligible. ${ }^{15}$ Absolute values of the linear term of the heat capacity, $\gamma$, 
taken under $P$ are accurate to $\pm 2 \mathrm{~mJ} / \mathrm{mol} \mathrm{V} \mathrm{K}^{2}$.

In order to determine the effective mass, $m^{*}$, from the heat capacity and resistivity measurements, we need to know the density of carriers, $n$. We plot in Fig. 1 the Hall coefficient $R_{H}$ versus $T$ for $\mathrm{V}_{1.987} \mathrm{O}_{3}$ under pressure and for a series of vanadium deficient samples, $\mathrm{V}_{2-y} \mathrm{O}_{3}$. Increasing pressure or increasing the number of vanadium vacancies drives the system more metallic with a critical pressure for $\mathrm{V}_{1.987} \mathrm{O}_{3}$ of $2.25 \pm 0.1 \mathrm{kbar}$ and a critical concentration of $y=0.015$. Between these temperatures, we observe a marked temperature dependence which is very similar to what is observed for heavy fermion systems, ${ }^{16}$ where $R_{H}(T)$ is attributed to incoherent magnetic skew scattering. ${ }^{17}$ For $\mathrm{V}_{2-y} \mathrm{O}_{3}$, the rise in $R_{H}$ with decreasing $T$ coincides with the appearance of magnetic peaks in the inelastic neutron scattering data, ${ }^{8}$ reaching a maximum at $T_{N}$ where spin fluctuations start to freeze out, consistent with the qualitative picture of carriers scattering off spin fluctuations.

A proper quantitative analysis of $R_{H}(T)$ is complicated since $\mathrm{V}_{2} \mathrm{O}_{3}$ has two occupied $d$ bands. Nonetheless, we can understand the pertinent behavior within a simple one-band picture. Analyzing our data in the temperature-independent regimes, we find that the density of holes remains constant with pressure, but increases linearly with $y$. Above $150 \mathrm{~K}$, all the pressure data sets merge to the same value of $1 / e R_{H}$ of $3.5(0.3) \times 10^{22}$ holes $/ \mathrm{cm}^{3}$, which is equivalent to 1 hole per $\mathrm{V}$ atom. The $\mathrm{V}_{2} \mathrm{O}_{3}$ system has 2 electrons $/ V^{3+}$ which, if they completely fill a low-lying nondegenerate $a_{1 g}$ band and half fill a higher lying doubly degenerate $e_{\pi}$ band, would yield exactly 1 carrier $/ \mathrm{V}$ atom. Therefore, $\mathrm{V}_{1.987} \mathrm{O}_{3}$ still appears to be very close to half-filling (a completely filled lower Hubbard band and an empty upper Hubbard band) until doped metallic with sufficient $\mathrm{V}$ vacancy concentration. Our values are similar to those reported by $\mathrm{McWhan}$ et al. for pure metallic $\mathrm{V}_{2} \mathrm{O}_{3}$ under pressure, where $R_{H}(T=4.2 \mathrm{~K})=3.5 \pm 0.4 \times 10^{-4} \mathrm{~cm}^{3} / \mathrm{C}$. ${ }^{18}$

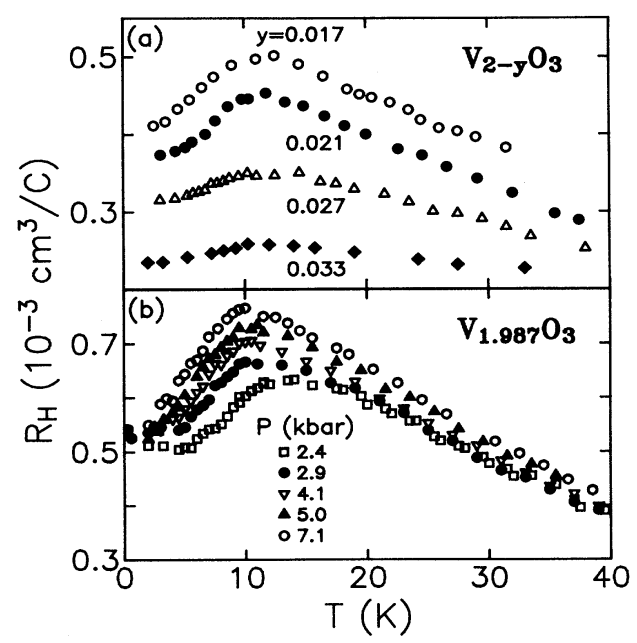

FIG. 1. Hall coefficient $R_{H}$ vs temperature $T$ for (a) a series of barely metallic $\mathrm{V}$ deficient crystals and (b) an insulating crystal driven metallic with pressure. $R_{H}$ (and hole density $n$ ) scale linearly with $y$, but are independent of $P$ as $T \rightarrow 0$.

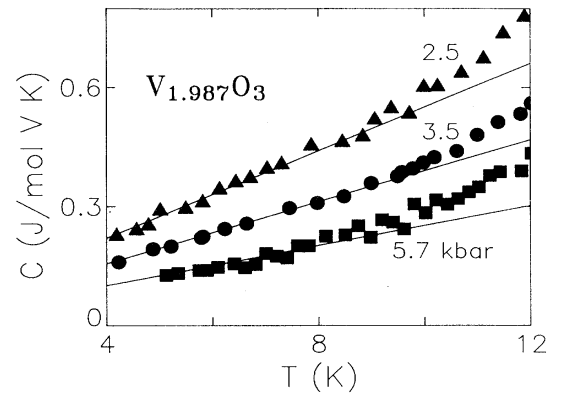

FIG. 2. Specific heat $C$ depends linearly on temperature at low $T$ for a series of pressures $P$, with increasing $\gamma$ (slope) at the approach to the MIT $\left(P_{c}=2.25 \mathrm{kbar}\right)$.

We now evaluate the behavior of the effective mass, $m^{*}$, as the MIT is approached from above with pressure. We show in Fig. 2 the heat capacity versus $T$ of $\mathrm{V}_{1.987} \mathrm{O}_{3}$ under $P$ between 4 and $12 \mathrm{~K}$. There is very little change in the entropy at the ordering temperature $T_{N} \sim 10 \mathrm{~K}$, indicating that the magnetic order is mostly itinerant. $\mathrm{Up}$ to $30 \mathrm{~K}$ there is no $T^{3}$ term characteristic of contributions from SDW order, magnons, or the lattice. The marked change in slope above $T_{N}$ implies that there is an additional contribution to the heat capacity which is likely of magnetic origin. Spin fluctuations, which are partially frozen out below $T_{N}$, have $C \propto T$ (Ref. 19) and are known to dominate over the SDW contribution in other systems. ${ }^{20} \mathrm{NMR}$ measurements show that $\mathrm{V}_{2-y} \mathrm{O}_{3}$ has a mixture of majority $V^{3+}$ sites and minority $\mathrm{V}^{4+}$ sites. $^{21}$ The relative number of minority and majority states changes with doping but remains constant with pressure. Thus, the contribution to the heat capacity from the spin fluctuations should be constant with pressure as reflected by the similar slopes above $T_{N}$.

The linear slope in $C(T)$ as $T \rightarrow 0, \gamma$, arising from electron-electron correlations is proportional to $m^{*} / n^{2 / 3}$. Given that $n$ is constant, the large increase in $\gamma$ which occurs with decreasing pressure must be due to an increase in the effective mass $m^{*}$. The effective mass clearly acts as if it is diverging as the first-order MIT is approached with pressure (Fig. 5). This Brinkman-Ricelike behavior has been observed recently in the solid solution perovskite $\mathrm{La}_{x} \mathrm{Sr}_{1-x} \mathrm{TiO}_{3}$ as a function of doping. ${ }^{22}$ However, to our knowledge, the $\mathrm{V}_{2-y} \mathrm{O}_{3}$ results are the first observation of such behavior both where the density of carriers has been fixed near half-filling and where magnetic order characteristic of important classes of highly correlated metals plays a prominent role.

The discovery of a diverging $m^{*}$ at the Mott-Hubbard transition in vanadium sesquioxide depends crucially on performing the appropriate experiments in the appropriate $T \rightarrow 0$ limit. If we were to analyze the canonical higher temperature characteristics such as the enhancement in the susceptibility or the $T^{2}$ dependence of the resistivity, ${ }^{11,22}$ then we would draw erroneous conclusions about the key physics at the MIT. The spin fluctuations and order demand a sea change in the traditional Brinkman-Rice approach to correlated metals, whether they be transition metal oxides, rare-earth oxides, or actinide compounds. We demonstrate this explicitly by graphing in Fig. 3 the $T^{2}$ behavior of the resistivity for 


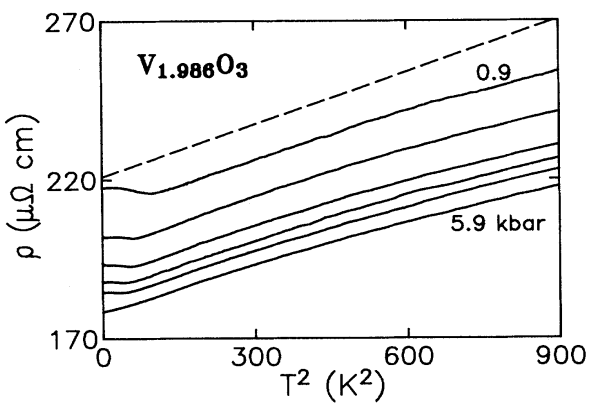

FIG. 3. Resistivity $\rho$ vs $T^{2}$ (a correlation term) for a series of pressures $\left(0.9,2.2,2.8,3.2,4.1,5.9 \mathrm{kbar}\right.$, with $\left.P_{c}=0.5 \mathrm{kbar}\right)$ does not reflect the diverging $\mathrm{m}^{*}$; it is likely dominated by spin fluctuations for these $T>T_{N}$. Dashed line has a $T^{2}$ slope of $0.55 \mu \Omega \mathrm{cm} / \mathrm{K}^{2}$ observed previously (Ref. 18 ).

$\mathrm{V}_{1.986} \mathrm{O}_{3}$ at a series of pressures, the slope being a timehonored measurement of an enhanced effective mass due to electron-electron interactions. The fit to $T^{2}$ is over a narrow range, being cut off by $T_{N}$ at the low end and deviating at the high end, but quantitatively consistent with previous measurements. ${ }^{18}$ Remarkably, the slope does not change as the MIT is approached, even though we know from our heat capacity measurements that $m^{*}$ is rapidly increasing over the same pressure interval. The large $T^{2}$ slope, in fact, simply reflects the existence of robust spin fluctuations, ${ }^{8}$ and the constancy of the slope reinforces our earlier conclusion that the spin fluctuation spectrum does not change with pressure. It is only at $T \ll T_{N}$ that the resistivity can be used to solve for the mass enhancement due to electron-electron correlations. Below $1 \mathrm{~K}$ the diffusive resistivity in the barely delocalized metal follows a $T^{1 / 2}$ temperature dependence, whose slope is a function of $m^{*}$ and $n .^{23}$ This slope indeed changes rapidly with $P$ in compressed $\mathrm{V}_{1.986} \mathrm{O}_{3},{ }^{4}$ tracking the heat capacity results.

We can gain more understanding of the role of spin fluctuations by studying the MIT as a function of doping, where we are changing the relative number of $V^{3+}$ and $V^{4+}$ sites. The Hall effect measurements (Fig. 1) have established already that the density of holes increases with doping. We show in the inset to Fig. 4 the heat capacity

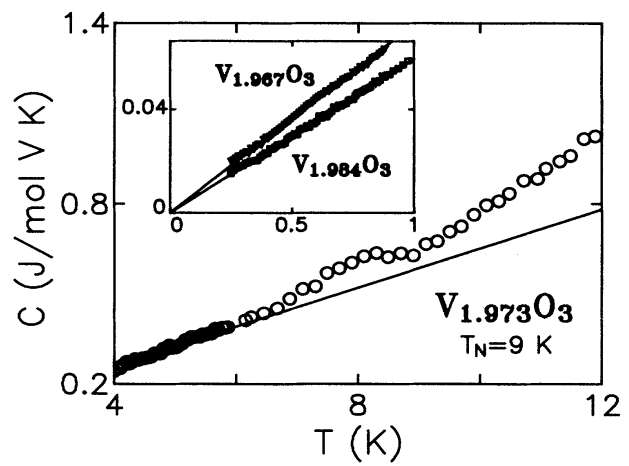

FIG. 4. Specific heat vs temperature showing change in slope and small change in entropy at $T_{N}$. Inset: Extension to low $T$, showing increasing $\gamma$ (slope) with increasing band-filling $(y)$, away from the MIT. in the $T \rightarrow 0$ limit for a series of $\mathrm{V}_{2-y} \mathrm{O}_{3}$ samples. The linear slope in $C(T), \gamma$, decreases as the MIT is approached. This result is exactly opposite to what we observed for the pressurized system, and, therefore, we conclude that the additional term in $C(T)$ due to the spin fluctuations must be strongly affected by doping. We graph in the main part of Fig. 4 the heat capacity of the $y=0.027$ sample up to $T=12 \mathrm{~K}$. The data is very similar to that for the pressurized system, but with better signal to noise without a background subtraction for the pressure cell. We now can see clearly the entropy change which occurs at $9 \mathrm{~K}$ due to the magnetic ordering. The excess entropy of the transition, $\Delta S=0.02 \mathrm{~J} / \mathrm{mol} \mathrm{V} \mathrm{K}$, is very small (an ordered moment $\mu=0.15 \mu_{B}$ has $\Delta S=0.43$ $\mathrm{J} / \mathrm{mol} \mathrm{VK}$ ), as expected for magnetism associated with itinerant carriers. The change in slope above $T_{N}$ is again due to an increase in the number of spin fluctuations.

We emphasize the difference between doping and pressure in correlated metals by plotting $\gamma$ versus $y$ and $P$ in Fig. 5. While $3 d$ band-filling additionally enhances $\gamma$, the compressed system appears to behave like a BrinkmanRice metal even in the presence of long-range antiferromagnetic order. We can analyze all of our data within a simple model by letting $\gamma=\gamma_{c}+\gamma_{s}$, where $\gamma_{c}$ is due to electron-electron interactions (Brinkman-Rice) and $\gamma_{s}$ is due to the spin fluctuations. The behavior of $\gamma_{c}$ is given by $\gamma_{c}=\gamma_{0}\left[1-\left(U / U_{c}\right)^{2}\right]^{-1}$, where $\gamma_{0}$ is the $\gamma$ expected for a noninteracting $V^{3+}$ in a band and $U$ is the intraatomic Coulomb interaction with value $U_{c}$ at the MIT. ${ }^{12}$ Our data is consistent with the assumptions that the ratio $U / U_{c}$ varies linearly with $P / P_{c}$ and that the spin fluctuation term $\gamma_{s}$ increases proportional to the doping $y$.

The solid lines in Fig. 5 show our best fits to the above model. We find that under pressure, where $\gamma_{s}$ is constant, the data obey a Brinkman-Rice-like form, and the electronic effective mass diverges at the Mott-Hubbard transition. The pressurized system can be understood within the usual picture of competition between the bandwidth $B$ and the repulsive Hubbard $U$. As pressure is increased, the bandwidth of the upper and lower Hubbard bands increases. When $B>U$, the system undergoes a MIT. The transition is parametrized by a discontinuous change in $n$ (from 0 to 1 hole/ $\mathrm{V}$ atom) and a diverging $m^{*}$. As the pressure is further increased, $m^{*}$ de-

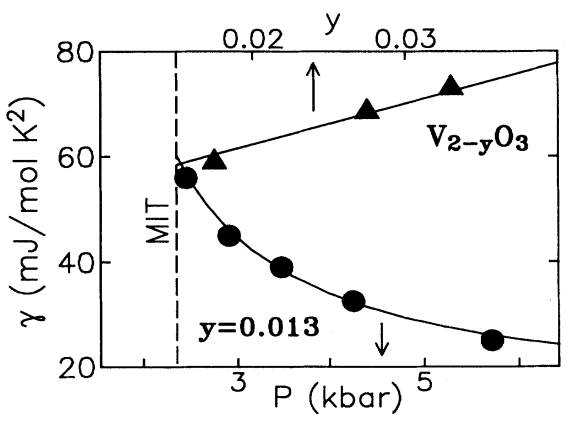

FIG. 5. $\gamma$ vs $\mathrm{V}$ vacancy concentration $y$ and pressure $P$. Combining this data with Hall data (Fig. 1), gives a diverging effective mass $m^{*}$ at the metal-insulator transition with $P$, but a decreasing $m^{*}$ with band-filling. Solid lines are fits to a model described in the text. 
creases and the number of doubly occupied sites, $\eta$, increases, leading to a reduction in $T_{N}$ and the dissolution of the long-range magnetic order. At sufficiently high $P$ the system undergoes a transition to a paramagnetic metal at all $T$, which should be the traditional BrinkmanRice system. The $T=0$ phase boundary between antiferromagnetic metal and paramagnetic metal indeed has been observed for pressurized $\mathrm{V}_{1.986} \mathrm{O}_{3}{ }^{4}$

The doped system clearly behaves much differently. We find a very large $\gamma_{c}$ of $47 \pm 3 \mathrm{~mJ} / \mathrm{mol} \mathrm{V} \mathrm{K}^{2}$, corresponding to $U / U_{c}=0.92$, which is constant with bandfilling. Given that $n$ increases with $y$, the effective mass must actually decrease slightly as the MIT is approached from above. The change in $\gamma$ with doping, therefore, is determined entirely by $\gamma_{s}$. Hence, the MIT cannot be driven by a change in the ratio of $B / U$ as in compressed $\mathrm{V}_{2-y} \mathrm{O}_{3}$. Rather, the data is consistent with the MIT being driven by deviations from half-filling (completely filled lower Hubbard band). Such a transition still would be marked by a discontinuous change in $n$, but $m^{*}$ does not necessarily increase at the approach to the MIT. The antiferromagnetism mandates a large electronic effective mass since a small $m^{*}$ would imply a large number of doubly occupied sites, which would be antithetical to magnetic order. This explains why doping does not significantly decrease $T_{N}$. Although unexpected, the behavior of $\gamma$ with $y$ is plausible for a Mott-Hubbard system doped away from half-filling, with characteristics reminiscent of the metallic heavy fermion compounds.

In summary, the $T=0$ approach to the Mott-Hubbard transition in the correlated metallic state of vanadium sesquioxide looks completely different as a function of pressure or band-filling. Under pressure, we have discovered a diverging $m^{*}$ at the MIT, even in the presence of a spiral SDW. With changing hole concentration, the metal remains highly correlated, but the MIT appears to be driven by deviations from half-filling. These results stress the importance of parametrizing the MIT in terms of $m^{*}$ and $n$ through thermodynamic and transport probes in the true $T \rightarrow 0$ limit. The properties of the itinerant antiferromagnetic metal are so strongly affected by spin fluctuations that traditional probes of $m^{*}$ (such as the $T^{2}$ term in the resistivity) fail for $T>T_{N}$. Similar results are to be expected for the normal state of heavy fermion and cuprate superconductors.

We are grateful to G. Zimanyi for helpful discussions. The work at the University of Chicago was supported by NSF Contract No. DMR92-04820. J.M.H. and J.S. were supported on MISCON Grant No. DE-FG0290ER45427.
${ }^{1}$ D. W. Hess, P. S. Riseborough, and J. L. Smith, Encyclopedia of Applied Physics (VCH, New York, in press).

${ }^{2}$ S-W. Cheong et al., Phys. Rev. Lett. 67, 1791 (1991), and references therein.

${ }^{3}$ Y. Ueda, K. Kosage, and S. Kachi, J. Solid State Chem. 31, 171 (1980); J. Dumas and C. Schlenker, J. Magn. Magn. Mater. 7, 252 (1978).

${ }^{4}$ S. A. Carter et al., Phys. Rev. Lett. 67, 3440 (1991).

$5 \mathrm{~J}$. A. Wilson, in The Metallic and Nonmetallic States of Matter, edited by P. P. Edwards and C.N.R. Rao (Taylor and Francis, London, 1985), p. 216.

${ }^{6}$ R. N. Bhatt and D. S. Fisher, Phys. Rev. Lett. 68, 3072 (1992), and references therein.

${ }^{7}$ B. I. Shraiman and E. D. Siggia, Phys. Rev: Lett. 62, 1564 (19989); C. Jayaprakash, H. R. Krishnamurthy and S. Sarker, Phys. Rev. B 40, 2610 (1989); B. Chakraborty et al., ibid. 42, 4819 (1990).

${ }^{8}$ W. Bao et al., Phys. Rev. Lett. 71, 766 (1993).

${ }^{9}$ N. F. Mott, Proc. Phys. Soc. London A 62, 416 (1949); J. Hubbard, Proc. R. Soc. London A 281, 401 (1964).

${ }^{10}$ D. B. McWhan, T. M. Rice, and J. P. Remeika, Phys. Rev. Lett. 23, 1384 (1969); D. B. McWhan et al., Phys. Rev. B 7, 3079 (1973).

${ }^{11}$ W. F. Brinkman and T. M. Rice, Phys. Rev. Lett. 27, 941
(1971).

12J. Spalek, M. Kokowski, and J. M. Honig, Phys. Rev. B 39, 4175 (1989); N. Kawakami and S-K. Yang, Phys. Rev. Lett. 65, 2309 (1990); X. Y. Zhang, M. J. Rozenberg, and G. Kotliar, ibid. 70, 1666 (1993); V. Janis, J. Masek, and D. Vollhardt, Z. Phys. B (to be published).

${ }^{13}$ H. R. Harrison, R. Aragon, and C. J. Sandberg, Mater. Res. Bull. 15, 571 (1980).

${ }^{14}$ S. A. Shivishankar et al., J. Electrochem. Soc. 128, 2472 (1981).

${ }^{15}$ S. A. Carter et al., Phys. Rev. B 43, 607 (1991).

${ }^{16}$ M. Hadzic-Leroux et al., Europhys. Lett. 1, 579 (1986); Y. Onuki et al., J. Phys. Soc. Jpn. 58, 2119 (1989).

${ }^{17}$ A. Fert and P. M. Levy, Phys. Rev. B 36, 1907 (1987).

${ }^{18}$ D. B. McWhan et al., Phys. Rev. B 7, 1920 (1973).

${ }^{19}$ T. Moriya, J. Magn. Magn. Mater. 14, 1 (1979); H. Hasegawa, J. Phys. Soc. Jpn. 38, 107 (1975).

${ }^{20}$ T. Shinkoda, K. Kumagai, and K. Asayama, J. Phys. Soc. Jpn. 46, 1753 (1979).

${ }^{21}$ Y. Ueda et al., Phys. Chem. Solids 39, 1281 (1978).

${ }^{22}$ Y. Tokura et al., Phys. Rev. Lett. 70, 2126 (1993).

${ }^{23}$ B. L. Altshuler, A. G. Aronov, and P. A. Lee, Phys. Rev. Lett. 44, 1288 (1980); T. F. Rosenbaum et al., ibid. 46, 568 (1981). 